\title{
Suppression of Ion Transport due to Long-Lived Subwavelength Localization by an Optical Lattice
}

\author{
Leon Karpa,${ }^{1}$ Alexei Bylinskii, ${ }^{1}$ Dorian Gangloff, ${ }^{1}$ Marko Cetina,,${ }^{1,2}$ and Vladan Vuletić ${ }^{1, *}$ \\ ${ }^{1}$ Department of Physics and Research Laboratory of Electronics, Massachusetts Institute of Technology, \\ Cambridge, Massachusetts 02139, USA \\ ${ }^{2}$ Institute of Quantum Optics and Quantum Information, Otto-Hittmair-Platz 1, A-6020 Innsbruck, Austria
}

(Received 29 March 2013; published 16 October 2013)

\begin{abstract}
We report the localization of an ion by a one-dimensional optical lattice in the presence of an applied external force. The ion is confined radially by a radio frequency trap and axially by a combined electrostatic and optical-lattice potential. Using a resolved Raman sideband technique, one or several ions are cooled to a mean vibrational number $\langle n\rangle=(0.1 \pm 0.1)$ along the optical lattice. We measure the average position of a periodically driven ion with a resolution down to $\lambda / 40$, and demonstrate localization to a single lattice site for up to $10 \mathrm{~ms}$. This opens new possibilities for studying many-body systems with long-range interactions in periodic potentials, as well as fundamental models of friction.
\end{abstract}

PACS numbers: $37.10 . V z$, 03.67.Lx, 37.10.Rs, 37.10.Ty

Time-dependent optical forces play an important role in quantum information processing with ions [1,2], as they provide a means to convert the strong Coulomb interaction into a state-dependent ion-ion interaction [3-12]. Time-independent periodic optical potentials (optical lattices) have been used to study anomalous ion diffusion [13], and have been proposed for various quantum computation and simulation schemes [14-18]. An ion chain in an optical lattice represents a many-body system with strong long-range interactions that is predicted to exhibit both classical and quantum phase transitions in the context of the Frenkel-Kontorova friction model [19-22].

Compared to neutral atoms, the optical trapping of ions is substantially more difficult: even weak stray electric fields produce strong Coulomb forces, while in deep optical potentials the associated linewidth broadening thwarts efficient Doppler cooling. Nonetheless, by superimposing a tightly focused optical running-wave dipole trap with a Paul trap and turning the latter off after loading, trapping of a single ion for a few axial vibration periods (a few milliseconds) has been achieved [17]. In one-dimensional optical lattices, trapping times of tens of microseconds [23], as well as subwavelength localization in the lattice on microsecond time scales, corresponding to a few vibration periods [24], have been reported very recently.

In this Letter we demonstrate lattice-assisted Raman cooling and the long-lived localization of an ion to a single lattice site even when a sizeable electric field of $1 \mathrm{~V} / \mathrm{m}$ is applied. Measuring the ion's center position with a resolution of $20 \mathrm{~nm}$, well below the $185 \mathrm{~nm}$ lattice period, we find that the electric-field-induced ion transport is fully suppressed by the lattice on time scales of up to $10 \mathrm{~ms}$, many orders of magnitude longer than the $\sim 1 \mu$ s oscillation period in the optical trap. We also demonstrate that a one-dimensional crystal of three ions can be cooled in the optical lattice, which will enable studies of classical and quantum friction [19-22] in the future.

The apparatus (see Fig. 1) is described in more detail in Ref. [25]. The planar Paul trap contains electrodes driven at a $16 \mathrm{MHz}$ radio frequency (rf), providing radial confinement with vibrational frequencies of $\omega_{z} \approx \omega_{x} \approx 2 \pi \times$ $1.1 \mathrm{MHz}$, while dc electrodes create electrostatic confinement along $y$. To generate a deep optical-lattice trap, we make use of an optical cavity (finesse $\mathcal{F} \approx 1500$ ) that is aligned with the linear rf quadrupole trap. The $\mathrm{TEM}_{00}$ mode with a waist size of $38 \mu \mathrm{m}$ is tuned into resonance with the lattice laser, which is blue-detuned by $\Delta_{1}=2 \pi \times$ 12.7 GHz from the ${ }^{2} S_{1 / 2} \rightarrow{ }^{2} P_{1 / 2}$ transition in ${ }^{174} \mathrm{Yb}^{+}$at a wavelength of $2 \pi / k=\lambda=369 \mathrm{~nm}$. The typical trap depth is $U / h=45 \mathrm{MHz}$, corresponding to a circulating power of $10 \mathrm{~mW}$ inside the cavity, and a lattice vibrational frequency of $\omega_{y}^{(\mathrm{OL})}=2 \pi \times 1.2 \mathrm{MHz}$.

The ion is prepared in a Paul trap with weak electrostatic $y$ confinement $\omega_{y}^{(\mathrm{dc})}=2 \pi \times 130 \mathrm{kHz}$, with the optical lattice superimposed. In order to optically cool the ion in the presence of ac Stark shifts of up to $2 U / h$ that exceed the atomic linewidth of $\Gamma=2 \pi \times 19.6 \mathrm{MHz}$ of the ${ }^{2} S_{1 / 2} \rightarrow$ ${ }^{2} P_{1 / 2}$ transition [26], we employ a lattice-assisted Raman sideband cooling method [27] where the trapping light itself drives Raman transitions [28,29]. As indicated in the inset of Fig. 1, the $\pi$-polarized trap light in combination with a circularly polarized beam $\sigma_{1}^{-}$along the $z$ axis drives Raman transitions between the levels $\left|{ }^{2} S_{1 / 2}, m=-1 / 2\right\rangle$ and $\left|{ }^{2} S_{1 / 2}, m=1 / 2\right\rangle$. The beam along $z$ is frequency shifted by $\delta=2 \pi \times 6 \mathrm{MHz}$ relative to the lattice laser, and a magnetic field along the $z$ axis is used to tune the Raman transition into resonance with the vibrational sideband $\mid m=$ $\left.-1 / 2 ; n_{y}, n_{z}\right\rangle \rightarrow\left|m=1 / 2 ; n_{y}-1, n_{z}\right\rangle$, where $n_{y}$ and $n_{z}$ indicate the trap vibrational levels in the $y$ and $z$ directions, respectively. In the blue-detuned lattice the atoms are 


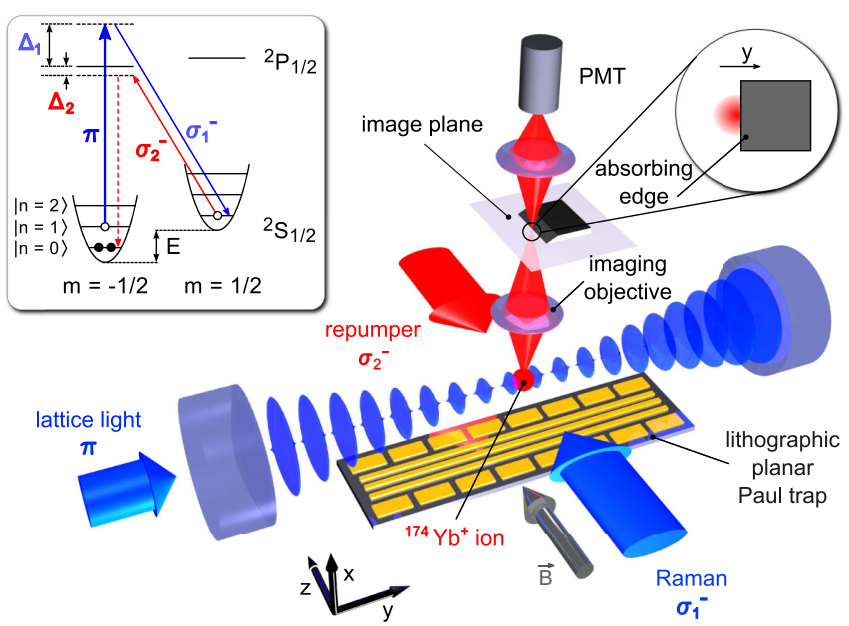

FIG. 1 (color online). Setup for trapping one or several ions in an optical lattice. An optical resonator is aligned with the linear Paul trap, located $135 \mu \mathrm{m}$ above the surface of a microchip. The inset shows the relevant transitions in ${ }^{174} \mathrm{Yb}^{+}$ions. The far-detuned $\pi$-polarized lattice and Raman $\sigma_{1}^{-}$beams drive a two-photon transition that reduces the ion's energy, while the near-resonant $\sigma_{2}^{-}$beam optically pumps the ion into its initial spin state $\left|{ }^{2} S_{1 / 2}, m=-1 / 2\right\rangle$, completing the cooling cycle. An absorbing edge in the image plane maps the ion fluorescence to position, enabling measurement resolution down to $\lambda / 40$.

trapped near the nodes where the vibrational Raman coupling is of the form $\left\langle n_{y}^{\prime}, n_{z}^{\prime}\left|e^{i k z} \sin (k y)\right| n_{y}, n_{z}\right\rangle$, so that the only cooling to first order in the Lamb-Dicke parameter $\eta=$ $\left(\omega_{\text {rec }} / \omega_{y}^{(\mathrm{OL})}\right)^{1 / 2}$ occurs along $y$, where $\omega_{\text {rec }}=2 \pi \times 8 \mathrm{kHz}$ is the recoil frequency. On this transition the Rabi frequency is $\Omega_{2} \sqrt{n} \eta$, where $\Omega_{2} \sim 2 \pi \times 2.5 \mathrm{MHz}$. To complete the cooling cycle, the ion is illuminated with a near-resonant $\sigma^{-}$-polarized pumping beam $\left(\sigma_{2}^{-}\right)$, detuned from the excited state by $\Delta_{2}=-2 \pi \times 100 \mathrm{MHz}$, that populates the $|m=-1 / 2\rangle$ state via spontaneous decay. Under ideal conditions, population accumulates in the dark lowest vibrational level $\left|m=-1 / 2 ; n_{y}=0\right\rangle$ that is coupled neither by the Raman transition nor by the optical pumping beam.

In the blue-detuned lattice the ac Stark shift at the potential minimum is zero, and increasing temperature along the lattice direction $(y)$ results in an ac Stark shift that reduces the detuning of the optical pumping beam $\left(\sigma_{2}^{-}\right)$from resonance, increasing the scattering. (This is illustrated in the inset of Fig. 2(a), where an ion positioned at different locations in the optical lattice by a strongly confining electrostatic potential along $y$ exhibits a spatial variation in fluorescence [25].) The scattering rate at the antinodes is significantly increased compared to the nodes. Consequently, in the optical lattice the ion's photon scattering rate is a measure of temperature along $y$, and can be used to identify Raman cooling.

Figure 2(a) shows ion fluorescence from a Raman cooled ion in an optical lattice. The spectrum is obtained by varying
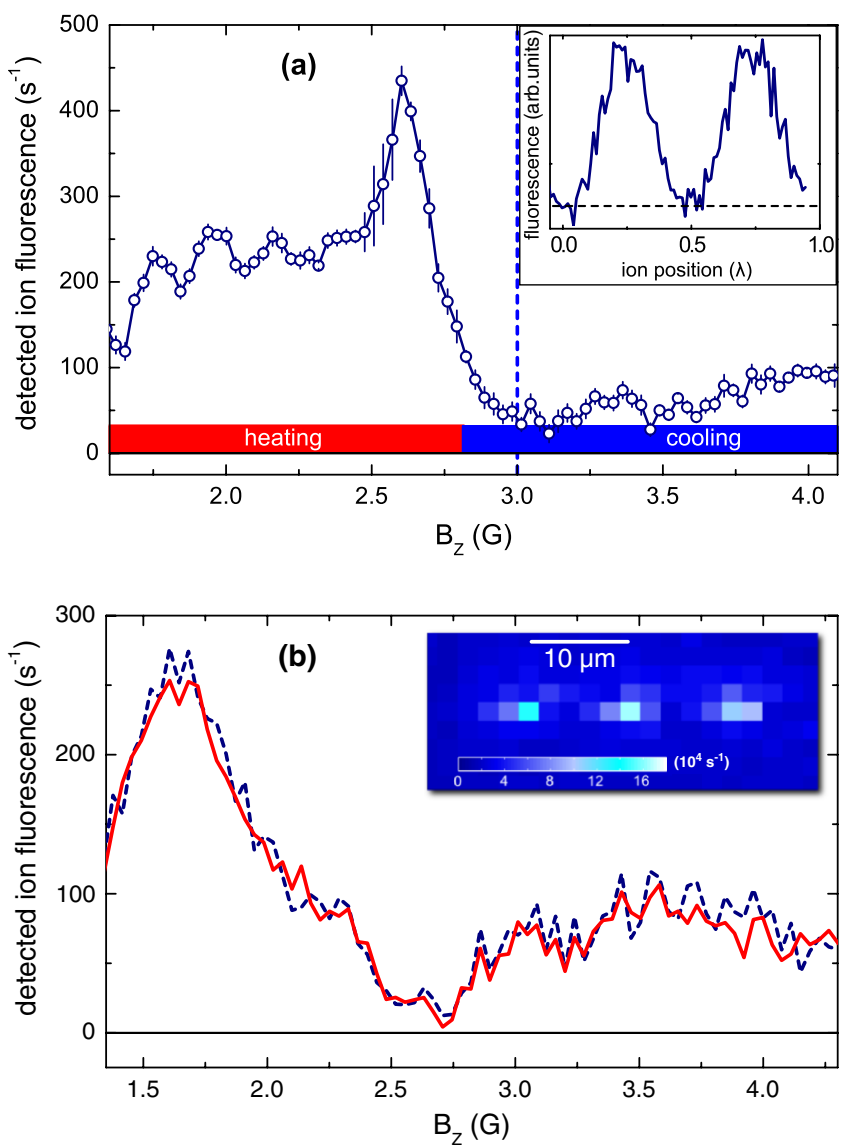

FIG. 2 (color online). (a) Fluorescence of a Raman cooled ion in an optical lattice with a depth of $U / h=(45 \pm 5) \mathrm{MHz}$ as a function of magnetic field $B_{z}$ that adjusts the two-photon detuning. The background due to light scattering from the chip has been subtracted. The vertical dashed line indicates the predicted red sideband transition at $(3.0 \pm 0.2) \mathrm{G}$. The variation in scattering rate originates from the temperature-dependent trapinduced ac Stark shift. The inset shows the ion florescence at different locations inside the cavity mode using a strongly confining electrostatic potential, where the dashed line indicates the scattering rate without the optical lattice. (b) Simultaneous Raman cooling of a three-ion crystal (as depicted in the inset under Doppler cooling conditions) in the optical lattice with average fluorescence per ion (solid) and center-ion fluorescence (dashed). The parameters for (a),(b) are $\Delta_{2} /(2 \pi)=$ $(-100,-120) \mathrm{MHz}$ and $\Omega_{2} /(2 \pi) \sim(2.5,12) \mathrm{MHz}$.

the magnetic field $B_{z}$ along $z$ in order to change the Raman resonance condition. On the heating side, where a Raman transition increases the ion's energy, the ion delocalizes in the optical lattice, which increases the scattering rate by the optical pumping beam. The principal cooling feature is the decrease of fluorescence almost to zero near $B_{z}=3 \mathrm{G}$ corresponding to the two-photon detuning $\delta_{R}=\mu_{B} B_{z} / \hbar-$ $\left(\delta+\Delta_{\text {ac }} / \hbar\right)=\omega_{y}^{(\mathrm{OL})}=2 \pi \times(1.2 \pm 0.1) \mathrm{MHz}$ expected for the red sideband of the Raman transition coupling the states $\left|m=-1 / 2 ; n_{y}\right\rangle$ and $\left|m=1 / 2 ; n_{y}-1\right\rangle$. Here, with $\mu_{B}$ being the Bohr magneton, $\Delta_{\mathrm{ac}} / \hbar \approx 2 \pi \times(0.7 \pm 0.1) \mathrm{MHz}$ 
at the node, $\mu_{B} B_{z} / \hbar$, and $\delta=2 \pi \times 6 \mathrm{MHz}$ denote the differential ac Stark shift between the ground states induced by the optical pumping beam, the Zeeman splitting of these states, and the frequency difference between the two Raman beams, respectively. Since any sizeable extent of the ion's wave function compared to the lattice period of $\lambda / 2=$ $185 \mathrm{~nm}$ would lead to an increase of fluorescence, as observed on the heating side $\delta_{R}<0$, the fluorescence dip at $\delta_{R}=\omega_{y}^{(\mathrm{OL})}$ can be interpreted as a signature of localization in a lattice site to a spatial extent $\ll \lambda / 2$. As Fig. 2(b) shows, the same method can be applied to a one-dimensional crystal consisting of three ions spaced by $\sim 10 \mu \mathrm{m}$, yielding a similar spectrum.

Under ideal conditions, the fluorescence rate due to Raman transitions is proportional to the population outside the dark state $\left|m=-1 / 2 ; n_{y}=0\right\rangle$, i.e., $\left\langle n_{y}\right\rangle /\left(\left\langle n_{y}\right\rangle+1\right)$. Hence, in the regime where $\Omega_{2} \sqrt{\left\langle n_{y}\right\rangle} \eta \gg \Gamma_{\text {op }}$, with $\Gamma_{\text {op }}=$ $(2.3 \pm 0.3) \times 10^{5} \mathrm{~s}^{-1}$ being the excitation rate, the residual observed fluorescence can be used to estimate $\left\langle n_{y}\right\rangle$. The fluorescence rate due to the optical pumping process is given by $3\left(\Gamma_{\mathrm{op}} / 2\right)\left\langle n_{y}\right\rangle /\left(\left\langle n_{y}\right\rangle+1\right)$, where the factor of 3 arises from the branching ratio of the spontaneous decay from $\left.{ }^{2} P_{1 / 2}, m=-1 / 2\right\rangle$ and the factor $1 / 2$ accounts for twophoton saturation. In this limit, we find $\left\langle n_{y}\right\rangle=(0.6 \pm 0.1)$ for the conditions of Fig. 2(a). After carefully minimizing residual micromotion we obtain $\left\langle n_{y}\right\rangle=(0.1 \pm 0.1)$ for one ion and $\left\langle n_{y}\right\rangle=(0.10 \pm 0.02)$ for the three-ion crystal, both consistent with ground-state cooling. (We attribute the observed heating in the presence of residual micromotion to finite spectral overlap between the vibrational levels broadened by cooling and the rf drive, resulting in off-resonant heating. Additionally, in the presence of both longitudinal and transverse micromotion, from the viewpoint of the ion, the Raman lasers acquire rf sidebands which lead to additional couplings and an increase in the ion fluorescence and temperature [30].) Thus the average axial energy of the cooled ion $\langle E\rangle / h=\left(1 / 2+\left\langle n_{y}\right\rangle\right) \omega_{y}^{(\mathrm{OL})} /(2 \pi)=$ $(0.7 \pm 0.1) \mathrm{MHz}$ is substantially below the optical-lattice depth of $U / h=45 \mathrm{MHz}$, confirming localization of the ion(s) well below the lattice spacing $\lambda / 2$.

Having established the condition for Raman cooling and ion localization in the lattice, we now proceed with timeresolved measurements of ion transport by a periodic applied electric field. This allows for direct probing of optical trapping on different time scales, and represents an enabling method for future studies of friction with ion chains $[19,20,22]$. In order to measure the ion position with a resolution below that of our imaging system of $D=2.9 \mu \mathrm{m}$, we position a sharp absorbing edge in the imaging plane such that it blocks half the ion fluorescence, as depicted in Fig. 1. The remaining light collected on a photomutiplier tube then linearly depends on the ion's position, with a measured fractional change of $2.5 \times$ $10^{-4} / \mathrm{nm}$, as calibrated with a charge-coupled camera.
By sufficiently long signal integration we can find the average ion position to much better than $D$ or even the optical wavelength $\lambda$ [31,32]. Since our photon detection rate at the optimum Raman cooling point is less than $30 \mathrm{~s}^{-1}$, and we need many photons for a sufficiently high signal-to-noise ratio, we employ a lock-in technique that enables resolving relative ion motion on time scales much shorter than the signal integration time. We also slightly misalign the polarization of the optical pumping beam $\sigma_{2}^{-}$ to increase the fluorescence to typically $100 \mathrm{~s}^{-1}$, which unfortunately also thwarts a temperature determination with the aforementioned method based on the residual fluorescence. In the Paul trap with low axial frequency $\omega_{y}^{(\mathrm{dc})}=2 \pi \times 130 \mathrm{kHz}$, we apply a slowly varying electric force with period $T$ to displace the ion along the $y$ axis, measure the time-resolved ion fluorescence synchronously with the applied force, and integrate the signal. We choose the amplitude of the driving field to displace the ion by $A \cong \pm 2$ lattice sites. This corresponds to a field amplitude of $0.7 \mathrm{~V} / \mathrm{m}$, which is an order of magnitude higher than the estimated slowly varying residual electric fields. The corresponding data under Doppler cooling conditions are shown in Fig. 3 as the blue solid squares. With an integration time of $150 \mathrm{~s}$, we can resolve the ion's response to the applied force down to $10 \mathrm{~nm} \approx \lambda / 40$. A related lock-in measurement technique of ion motion in the frequency domain yields a similar resolution [32].

We then turn on the optical lattice and Raman cooling. If the modulation is not too slow, we observe a strong suppression of the ion's driven motion [see Fig. 3(a)], to an amplitude consistent with zero $A^{(\mathrm{OL})}=(10 \pm 20) \mathrm{nm}$, and much smaller than the lattice spacing $\lambda / 2=185 \mathrm{~nm}$ or the amplitude $A=(340 \pm 10) \mathrm{nm}$ in the pure Paul trap under the same conditions. For an ion confined to a single site, the expected amplitude is $4 \mathrm{~nm}$ in the lattice with vibration frequency $\omega_{y}^{(\mathrm{OL})}=2 \pi \times 1.2 \mathrm{MHz}$, consistent with our observation. Similar results are obtained for driving periods $T$ between $31 \mu$ s and $10 \mathrm{~ms}$. We conclude that for up to $10^{4}$ vibration periods, the ion remains confined to a single lattice site. At still lower driving frequencies ( $T \gtrsim 10 \mathrm{~ms}$ ) the continuously cooled ion has some finite probability to thermally hop across the lattice potential barrier during the observation time, resulting in a partial suppression of the driven motion when averaged over many trials. This partial amplitude suppression is accompanied by a delay in the ion's motion with respect to the driving force [see Fig. 3(b)].

In order to compare the suppression of the ion transport by the lattice to a model, we measure the amplitude of the driven ion motion for different drive frequencies. Figure 4 shows the best repeatedly observed amplitude suppression for each frequency, from which we infer an approximate trapping time on the order of $0.1 \mathrm{~s}$, corresponding to an amplitude suppression by $30 \%$. In order to model the ion transport dynamics in the lattice, we assume that the ion's 


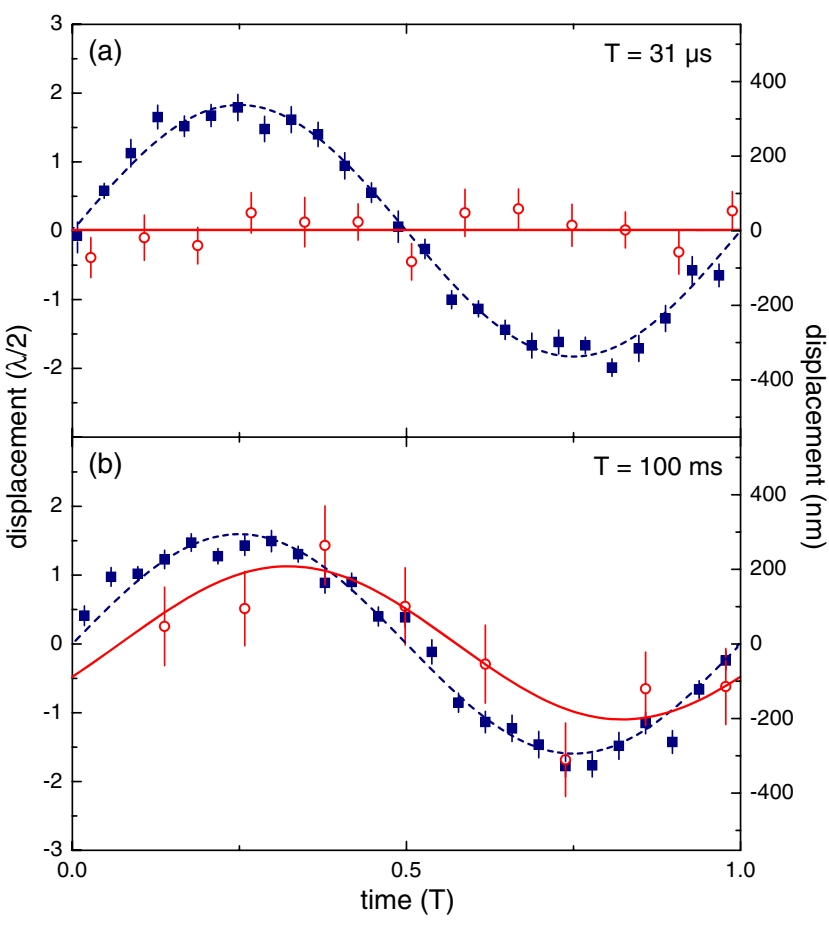

FIG. 3 (color online). Ion displacement by a periodic electric field in units of $\lambda / 2=185 \mathrm{~nm}$, without optical confinement (squares) and in an optical lattice (circles). (a) The modulation with a period of $T=31 \mu$ s was applied for averaging durations of 150 and $300 \mathrm{~s}$, respectively. The lines represent sinusoidal fits yielding amplitudes of $A=(340 \pm 10) \mathrm{nm}$ in the Paul trap and $A^{(\mathrm{OL})}=(10 \pm 20) \mathrm{nm}$ in presence of the optical lattice, respectively. (b) Corresponding measurements for a modulation period of $T=100 \mathrm{~ms}$. The fit yields $A=(300 \pm 10) \mathrm{nm}$, $A^{(\mathrm{OL})}=(200 \pm 40) \mathrm{nm}$, and a phase delay $\Delta \phi=\left(25^{\circ} \pm 11^{\circ}\right)$ of the driven motion in the lattice.

energy samples by a random walk the thermal distribution of the energies at a finite constant temperature. With a probability per unit time fixed by the cooling rate, the ion energy either increases or decreases by a motional quantum, weighted by the ratio of Boltzmann factors. At any given time, if the ion's energy is smaller than the effective depth of the lattice site (modified by the time-dependent external force), the ion stays localized at that site. Otherwise the ion follows the external displacement in the shallow electric potential, until its recapture occurs in one of the optical-lattice sites spanned by the ion's thermal distribution with accordingly weighted probabilities. As indicated by the solid line in Fig. 4, we find that both the characteristic trapping time and the approximate frequency dependence of the suppression of ion transport in the experiment are in good agreement with the results of the numerical simulation for a temperature of $250 \mu \mathrm{K}$, corresponding to a mean vibrational quantum number $\left\langle n_{y}\right\rangle=4$. The increase compared to $\left\langle n_{y}\right\rangle=0.1$ obtained from the Raman spectrum can be explained by the decrease in optical pumping efficiency due to the polarization

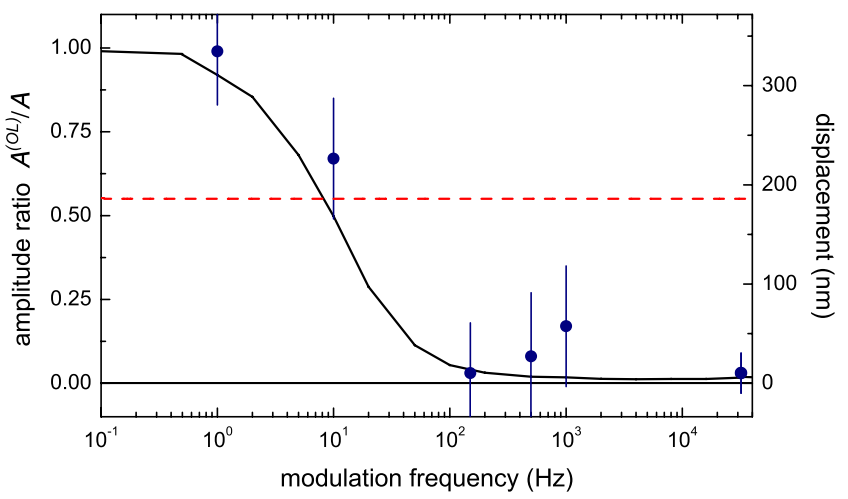

FIG. 4 (color online). Normalized ion oscillation amplitude in the lattice $A^{(\mathrm{OL})} / A$ as a function of the modulation frequency. The dashed line represents a displacement of $\lambda / 2$. The results of a numerical simulation for an optical pumping rate of $\Gamma_{\text {op }}=0.1 \Gamma$ and a temperature $T \approx 250 \mu \mathrm{K}$ are shown as a solid line.

misalignment that we have introduced to increase the signal-to-noise ratio of the ion position measurement. This increases the recoil heating and is expected to result in a higher temperature, in qualitative agreement with the numerical simulation.

In summary, we have demonstrated Raman cooling of up to three ions in an optical lattice close to the ground state, a lock-in method for detecting ion position with a resolution of $\lambda / 40$, and trapping of the ion at a single lattice site for many vibration periods even in the presence of an applied force. These results open various possibilities: the optical trapping may enable a strongly confining hybrid electrostatic-optical trap by combining electrostatic forces in two directions with the optical lattice in the third, thereby eliminating the driven micromotion in Paul traps that is disruptive to controlled ion-atom collisions [33-36]. Furthermore, the position detection may enable the study of friction models and of structural phase transitions of ion chains in periodic optical potentials $[19,20]$.

We thank Yufei Ge, Isaac Chuang, and Karl Berggren for assistance with the micorochip fabrication. This work was supported by the ARO and the NSF. L.K. gratefully acknowledges support by the Alexander von Humboldt Foundation, and A. B. and D. G. gratefully acknowledge support by the NSERC. L. K., A. B., and D. G. contributed equally to this work.

*vuletic@mit.edu

[1] D. J. Wineland, Nobel Prize in Physics in 2012.

[2] D. Leibfried, R. Blatt, C. Monroe, and D. Wineland, Rev. Mod. Phys. 75, 281 (2003).

[3] C. Monroe, D. M. Meekhof, B. E. King, W. M. Itano, and D. J. Wineland, Phys. Rev. Lett. 75, 4714 (1995).

[4] C. Roos, T. Zeiger, H. Rohde, H. C. Nägerl, J. Eschner, D. Leibfried, F. Schmidt-Kaler, and R. Blatt, Phys. Rev. Lett. 83, 4713 (1999). 
[5] D. Porras and J. I. Cirac, Phys. Rev. Lett. 93, 263602 (2004).

[6] A. Friedenauer, H. Schmitz, J. T. Glueckert, D. Porras, and T. Schaetz, Nat. Phys. 4, 757 (2008).

[7] R. Blatt and D. Wineland, Nature (London) 453, 1008 (2008).

[8] H. Häffner, C. Roos, and R. Blatt, Phys. Rep. 469, 155 (2008).

[9] K. Kim, M.-S. Chang, S. Korenblit, R. Islam, E. E. Edwards, J. K. Freericks, G.-D. Lin, L.-M. Duan, and C. Monroe, Nature (London) 465, 590 (2010).

[10] T. Monz, P. Schindler, J. T. Barreiro, M. Chwalla, D. Nigg, W. A. Coish, M. Harlander, W. Hänsel, M. Hennrich, and R. Blatt, Phys. Rev. Lett. 106, 130506 (2011).

[11] R. Islam, E. Edwards, K. Kim, S. Korenblit, C. Noh, H. Carmichael, G.-D. Lin, L.-M. Duan, C.-C. Joseph Wang, J. Freericks et al., Nat. Commun. 2, 377 (2011).

[12] R. Blatt and C. F. Roos, Nat. Phys. 8, 277 (2012).

[13] H. Katori, S. Schlipf, and H. Walther, Phys. Rev. Lett. 79, 2221 (1997).

[14] J. I. Cirac and P. Zoller, Nature (London) 404, 579 (2000).

[15] D. Porras and J. I. Cirac, Phys. Rev. Lett. 92, 207901 (2004).

[16] R. Schmied, T. Roscilde, V. Murg, D. Porras, and J. I. Cirac, New J. Phys. 10, 045017 (2008).

[17] C. Schneider, M. Enderlein, T. Huber, and T. Schaetz, Nat. Photonics 4, 772 (2010).

[18] C. Schneider, D. Porras, and T. Schaetz, Rep. Prog. Phys. 75, 024401 (2012).

[19] T. Pruttivarasin, M. Ramm, I. Talukdar, A. Kreuter, and H. Häffner, New J. Phys. 13, 075012 (2011).

[20] A. Benassi, A. Vanossi, and E. Tosatti, Nat. Commun. 2, 236 (2011).
[21] I. García-Mata, O. V. Zhirov, and D. L. Shepelyansky, Eur. Phys. J. D 41, 325 (2007).

[22] B. Hu and B. Li, Physica (Amsterdam) 288A, 81 (2000).

[23] M. Enderlein, T. Huber, C. Schneider, and T. Schaetz, Phys. Rev. Lett. 109, 233004 (2012).

[24] R. B. Linnet, I. D. Leroux, M. Marciante, A. Dantan, and M. Drewsen, Phys. Rev. Lett. 109, 233005 (2012).

[25] M. Cetina, A. Bylinskii, L. Karpa, D. Gangloff, K. M. Beck, Y. Ge, M. Scholz, A. T. Grier, I. Chuang, and V. Vuletić, New J. Phys. 15, 053001 (2013).

[26] S. Olmschenk, D. Hayes, D. N. Matsukevich, P. Maunz, D. L. Moehring, K. C. Younge, and C. Monroe, Phys. Rev. A 80, 022502 (2009).

[27] C. Monroe, D. M. Meekhof, B.E. King, S. R. Jefferts, W. M. Itano, D. J. Wineland, and P. Gould, Phys. Rev. Lett. 75, 4011 (1995).

[28] V. Vuletić, C. Chin, A. J. Kerman, and S. Chu, Phys. Rev. Lett. 81, 5768 (1998).

[29] A. J. Kerman, V. Vuletić, C. Chin, and S. Chu, Phys. Rev. Lett. 84, 439 (2000).

[30] J. I. Cirac, L. J. Garay, R. Blatt, A. S. Parkins, and P. Zoller, Phys. Rev. A 49, 421 (1994).

[31] M. Karski, L. Förster, J. M. Choi, W. Alt, A. Widera, and D. Meschede, Phys. Rev. Lett. 102, 053001 (2009).

[32] P. Bushev, G. Hétet, L. Slodička, D. Rotter, M. A. Wilson, F. Schmidt-Kaler, J. Eschner, and R. Blatt, Phys. Rev. Lett. 110, 133602 (2013).

[33] A. T. Grier, M. Cetina, F. Oručević, and V. Vuletić, Phys. Rev. Lett. 102, 223201 (2009).

[34] C. Zipkes, S. Palzer, L. Ratschbacher, C. Sias, and M. Köhl, Phys. Rev. Lett. 105, 133201 (2010).

[35] S. Schmid, A. Härter, and J. H. Denschlag, Phys. Rev. Lett. 105, 133202 (2010).

[36] M. Cetina, A. T. Grier, and V. Vuletić, Phys. Rev. Lett. 109, 253201 (2012). 